\title{
ORIGINAL ARTICLE MicroRNA signatures in B-cell lymphomas
}

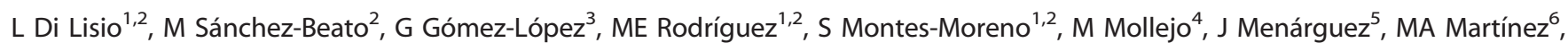 \\ FJ Alves ${ }^{7}$, DG Pisano ${ }^{3}$, MA Piris ${ }^{1,2,8}$ and N Martínez ${ }^{1,2,8}$
}

Accurate lymphoma diagnosis, prognosis and therapy still require additional markers. We explore the potential relevance of microRNA (miRNA) expression in a large series that included all major B-cell non-Hodgkin lymphoma (NHL) types. The data generated were also used to identify miRNAs differentially expressed in Burkitt lymphoma (BL) and diffuse large B-cell lymphoma (DLBCL) samples. A series of $147 \mathrm{NHL}$ samples and 15 controls were hybridized on a human miRNA one-color platform containing probes for 470 human miRNAs. Each lymphoma type was compared against the entire set of NHLs. BL was also directly compared with DLBCL, and 43 preselected miRNAs were analyzed in a new series of routinely processed samples of $28 \mathrm{BLs}$ and $43 \mathrm{DLBCLs}$ using quantitative reverse transcription-polymerase chain reaction. A signature of 128 miRNAs enabled the characterization of lymphoma neoplasms, reflecting the lymphoma type, cell of origin and/or discrete oncogene alterations. Comparative analysis of BL and DLBCL yielded 19 differentially expressed miRNAs, which were confirmed in a second confirmation series of 71 paraffin-embedded samples. The set of differentially expressed miRNAs found here expands the range of potential diagnostic markers for lymphoma diagnosis, especially when differential diagnosis of $B L$ and $D L B C L$ is required.

Blood Cancer Journal (2012) 2, e57; doi:10.1038/bcj.2012.1; published online 17 February 2012

Keywords: microRNA; B-cell lymphoma; microarray; miRNA expression profile; lymphoma diagnosis

\section{INTRODUCTION}

B-cell non-Hodgkin lymphomas (NHLs) are a group of lymphoproliferative B-cell disorders that include Burkitt lymphoma (BL), chronic lymphocytic leukemia (CLL), diffuse large B-cell lymphoma (DLBCL), mantle cell lymphoma (MCL), follicular lymphoma (FL), marginal zone lymphoma/mucosa-associated lymphoid tissue lymphoma (MZL/MALT), nodal marginal zone B-cell lymphoma (NMZL), splenic marginal zone lymphoma (SMZL) and various less frequent entities. ${ }^{1}$ These definitions are based on a combination of clinical data and morphological, phenotypic and cytogenetic features. The use of surrogate markers for gene and chromosomal alterations specific to the different B-cell lymphoma types ${ }^{1}$ is also useful at diagnosis.

Nevertheless, the distinctions between these disorders are somewhat blurred and most exhibit significant clinical and molecular heterogeneity. ${ }^{1,2} \mathrm{New}$, consistent markers continue to be required to improve the accuracy of lymphoma diagnosis and therapy selection. An area of particular interest is the interface between $\mathrm{BL}$ and $\mathrm{DLBCL},{ }^{3,4}$ two different lymphoma types that require different treatment. Previous studies have demonstrated the value of C-MYC translocations ${ }^{3}$ and gene expression profiling data ${ }^{4}$ for this purpose, but new markers are needed to delineate the boundaries between these two entities and to use this knowledge to identify diagnostic markers.

In recent years, the study of a new type of non-coding small RNA, microRNA (miRNA), has given renewed impetus to cell differentiation and cancer pathogenesis studies. ${ }^{5}$ MiRNAs post-transcriptionally regulate the expression of thousands of genes, including key genes in cell differentiation and cancer pathogenesis. ${ }^{6}$ Since evidence of the relationship between miRNAs and cancer first emerged, with the description of the loss of miR-15/16 in CLL cases, ${ }^{7}$ an increasing number of specific miRNA changes have been identified in many tumor types. ${ }^{5}$ The diagnostic potential of miRNAs is linked to their role in cellular differentiation, as demonstrated in hematopoietic cells and, for instance, by miR-150, in B-cell differentiation. ${ }^{8}$ The potential of using miRNAs for differential diagnosis of tumors and hematopoietic malignancies has been recognized, for example, in acute lymphoblastic and myeloid leukemias, where the expression signatures of at least two of four miRNAs (miR-128a, miR-128b, miR-223 and let-7b) distinguished the two tumor types with about $95 \%$ accuracy. ${ }^{9}$ Altered miRNA expression has a role in lymphoma development and potential diagnosis. MiRNA changes are associated with CLL (loss of miR-15a/16) ${ }^{7}$ and SMZL (loss of miR-29a and miR-29b-1), ${ }^{10}$ among others, and in both cases these miRNAs were located in frequently lost chromosomal regions: $13 q 14$ and $7 q 32$, respectively. There are differential signatures between the expression signature of patients with different lymphoma types, such as DLBCL and FL. ${ }^{11}$ Furthermore, in DLBCL, miRNAs are differentially expressed in the germinal center (GC) and activated B-cell-like (ABC) subtypes; in particular, miR-21, miR-155 and miR-221 were found to be upregulated in the $A B C$ subgroup. ${ }^{12,13}$

The purpose of this study is to test whether different B-cell lymphoma types have specific miRNA signatures, and to apply this knowledge to identify potential markers for distinguishing $\mathrm{BL}$ and DLBCL. In addition, the markers described here could be therapeutic targets in the treatment of B-cell lymphomas.

${ }^{1}$ Genomica del Cancer, Instituto de Investigación y Formación Marqués de Valdecilla (IFIMAV), Santander, Spain; ${ }^{2}$ Molecular Pathology Programme, Spanish National Cancer Research Centre (CNIO), Madrid, Spain; ${ }^{3}$ Structural Biology and Biocomputing Programme, Bioinformatics Unit, Spanish National Cancer Research Centre (CNIO), Madrid, Spain; ${ }^{4}$ Department of Pathology Hospital Virgen de la Salud, Toledo, Spain; ${ }^{5}$ Department of Pathology, HGUGM, Madrid, Spain; ${ }^{6}$ Department of Pathology, Hospital Universitario 12 de Octubre, Madrid, Spain and ${ }^{7}$ Department of Pathology, Hospital Universitario La Paz, Madrid, Spain. Correspondence: Dr N Martínez, Genomica del Cancer, Instituto de Investigación y Formación Marqués de Valdecilla (IFIMAV), Avda de Cardenal Herrera Oria S/N, 39011 Santander, Spain.

E-mail: ifimav.nmartinez@fmdv.org

${ }^{8}$ Senior authors.

Received 21 October 2011; revised 11 January 2012; accepted 13 January 2012 


\section{MATERIALS AND METHODS}

Sample selection

A series of 147 fresh-frozen samples of NHL, including $12 \mathrm{BL}, 29 \mathrm{DLBCL}, 22$ MCL, $17 \mathrm{SMZL}, 18 \mathrm{CLL}, 23 \mathrm{FL}, 11 \mathrm{NMZL}$ and $15 \mathrm{MZL} / \mathrm{MALT}$, and 15 nontumoral samples (7 reactive lymph nodes, 4 tonsils and 4 spleens) were collected. A formalin-fixed, paraffin-embedded (FFPE) series of 28 sporadic $\mathrm{BL}$ and 43 DLBCL samples were also investigated. Finally, an independent series of FFPE samples corresponding to 66 lymphomas (8 BL, $8 \mathrm{CLL}$, $12 \mathrm{DLBCL}, 9 \mathrm{FL}$, $8 \mathrm{MZL} / \mathrm{MALT}, 8 \mathrm{MCL}, 8 \mathrm{NMZL}$ and $5 \mathrm{SMZL}$ ) and 8 controls (4 reactive lymph nodes, 3 tonsils and 1 spleen) was examined by quantitative real-time-polymerase chain reaction (qRT-PCR) for miRNA validation. CLL samples correspond to lymph node-involved CLL cases to facilitate comparison with the other lymphoma types. All cases were reviewed by a panel of three hematopathologists (SMRP, MAP, SMM) according to the current World Health Organization criteria. ${ }^{1}$ All samples were diagnostic, taken before the patient received therapy. The project was approved by the ethics committee of the Instituto de Salud Carlos III (ISCIII). The majority of the BL cases (11/12 frozen cases; 20/28 FFPE cases) presented C-MYC translocation by FISH, whereas 31.7\% (13/41) of DLBCL FFPE cases were positive for C-MYC translocation. On the other hand, $60 \%$ $(15 / 25)$ and $40 \%(10 / 25)$ of DLBCL frozen samples were classified as $A B C$ and GC type, respectively, according to the classifier of Wright et al. ${ }^{14}$ based on gene expression profiling data, with four cases considered as not evaluable. FFPE samples of these cases permitted classification in $44 \%$ nonGC $(11 / 25)$ and $56 \%$ GC $(14 / 25)^{15}$ based on Hans immunohistochemical classifier. ${ }^{16}$ Finally, FFPE series of DLBCL used for qRT-PCR were classified as $54.76 \%$ non-GC (23/42) and $45.24 \%$ GC (19/42) (one case was not evaluable).

\section{MiRNA detection}

RNA from fresh-frozen tissues was extracted with Trizol reagent (Invitrogen, Carlsbad, CA, USA). Quality was assessed using a bioanalyzer (Agilent Technologies Inc., Santa Clara, CA, USA), and $100 \mathrm{ng}$ of total RNA were hybridized on an Agilent $8 \times 15 \mathrm{~K}$ Human miRNA one-color platform (Agilent Technologies Inc.) containing probes for 470 human miRNAs according to the manufacturer's guidelines. ${ }^{17}$ Data were extracted by Feature Extraction software (Agilent Technologies).
For FFPE samples, RNA was extracted by phenol-chloroform standard protocol after a deparaffinization step. RNA quality was assessed taking into account 260/280 ratio and 260/230 ratio. QRT-PCR was performed for 60 selected miRNAs according to the manufacturer's protocol (Applied Biosystems, Foster City, CA, USA)

\section{Statistical methods}

Between-array median normalization was carried out and significantly deregulated miRNAs were computed using the Significant Analysis of Microarray (SAM; http://www-stat.stanford.edu/ tibs/SAM/) method.

Each lymphoma type was compared against the whole set of samples. ${ }^{18}$ BL was also directly compared with DLBCL. MiRNAs with a false discovery rate (FDR) $<0.01$ and a $>1.5$-fold $\left(\log _{2}\right)$ change were considered to be significantly up- or downregulated between lymphoma types.

MiRNA target prediction was performed using the software analysis tools included into the following websites: http://gencomp.bio.unipd.it/ magia/start (Magia), http://www.targetscan.org (targetscan) and http:// diana.cslab.ece.ntua.gr/pathways (Diana Lab).

The K-nearest neighbors method was also used for class recognition (http://tnasas.bioinfo.cnio.es) to test whether these lymphomas were classified correctly with the selected miRNAs.

Quantitative RT-PCR data were processed using the SDS 2.2 and RealTime StatMiner (Integromics, Granada, Spain) programs, and normalized according to the most stable endogenous small RNA tested (RNU6B). $-\Delta \mathrm{Ct}$ values of $\mathrm{BL}$ and $\mathrm{DLBCL}$ were compared using a $t$-test (limma; available at http://pomelo2.bioinfo.cnio.es). MiRNAs with an FDR $<0.05$ were considered differentially expressed in the two groups. All clusters were computed using the web resource GEPAS 4.0 (www.gepas.es). The workflow is shown in Figure 1

\section{RESULTS}

MiRNA signature of B-cell NHLs

MiRNA expression profiles of $147 \mathrm{NHL}$ samples were studied. A general view of the B-cell lymphoma miRNA signature is shown in Supplementary Figure S1 comparing miRNA expression in tumor samples with non-tumoral controls (tonsils and lymph

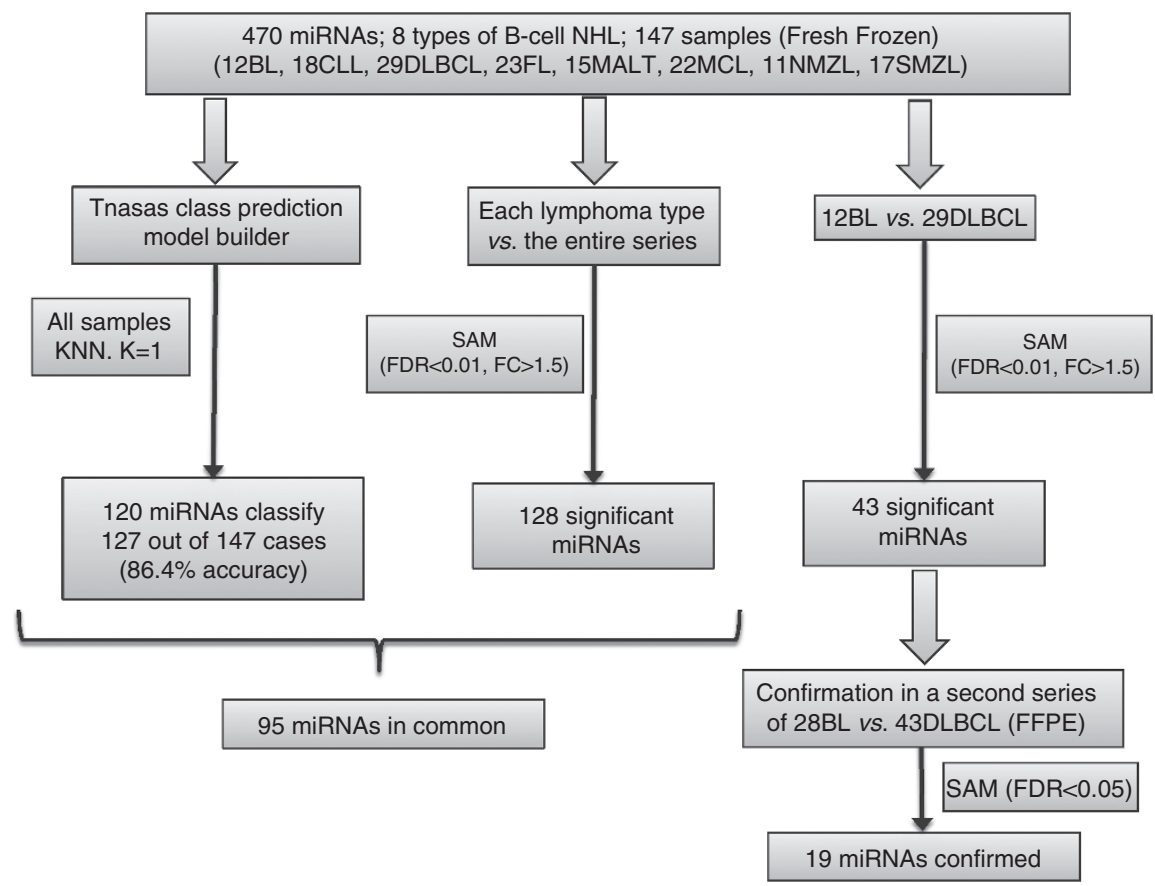

Figure 1. Workflow. The samples were analyzed first by SAM, comparing each lymphoma type against the whole series. In parallel, a class predictor model was investigated for the 147 samples. Also, BL and DLBCL samples were compared by SAM, and then significant miRNAs were analyzed in a second series of $B L$ and DLBCL samples. 
nodes or spleens). The heatmap reveals a fairly homogeneous expression pattern with a larger set of downregulated miRNAs in tumor cells. The upregulated miRNAs have a more heterogeneous pattern that varies with the lymphoma diagnosis.

The most strongly upregulated miRNAs compared with non-tumoral controls were miR-212, miR-487b, miR-513 and miR-770-5p, a set of miRNAs about which relatively little is known. On the other hand, downregulated miRNAs were more frequently observed. There was notable downregulation of let-7 family miRNAs, which are downregulated in various types of cancer, and key regulators of cell differentiation and apoptosis. ${ }^{19}$ Interestingly, let-7a and let-7c loss participates in the genesis and maintenance of the lymphoma phenotype in BL cells through C-MYC regulation. ${ }^{20}$ On the other hand, miR-23b was reported to be downregulated by C-MYC, playing a role in the MYC regulation of glutamine metabolism, and energy and reactive oxygen species homeostasis. ${ }^{21}$ The miR-200 family is another large family downregulated in this B-cell lymphoma series. MiR-200 has been described, along with the let-7 family, as a key regulator of cell differentiation, whose loss is associated with increased stemness capacity. ${ }^{19}$ MiR-10 (a and b) was also downregulated. This family is situated within the Hox cluster, and is also downregulated in myeloproliferative disorders. ${ }^{22}$ MiR-15 and miR-16, already described to be downregulated in $\mathrm{CLL}^{7}$ were found to be downregulated in the CLL cases in this study. MiR-155, which is involved in the immune response and GC development, and is upregulated in Hodgkin lymphoma, ${ }^{23}$ was found downregulated here in the $\mathrm{BL}$ cases.

A selection of 14 deregulated miRNAs ( 9 downregulated and 5 upregulated) was analyzed by qRT-PCR to validate the microarray data further. For this purpose, an independent series containing 66 lymphoma samples (8 CLL, 8BL, $12 \mathrm{DLBCL}, 9 \mathrm{FL}, 8 \mathrm{MZL} / \mathrm{MALT}$, $8 \mathrm{MCL}, 8 \mathrm{NMZL}$ and $5 \mathrm{SMZL}$ ) and 8 controls (4 lymph nodes, 3 tonsils and 1 spleen) was used.

The miRNA expression observed by microarrays was confirmed by qRT-PCR (see Supplementary Figure S4). Nevertheless, miR-487b, miR-212 and miR-770-5p presented a slight variability among the different lymphoma types, especially SMZL and MZL/ MALT. This variability can be explained by the different localization of SMZL lymphomas and NMZL, as SMZLs are localized in the spleen and MZL/MALT lymphomas are localized in a variety of different tissues (in this series: breast, eye, skin, salivary gland and intestine).

To select a lymphoma miRNA signature, data from all tumor samples, without previous normalization to controls, were studied. SAM analysis enabled comparison of each lymphoma type with the entire set of samples. A set of 128 miRNAs was considered to be significantly deregulated (FDR $<0.01 ;>1.5$-fold change in $\log _{2}$ ) in one or more lymphoma types (Table 1). The heatmap of the 128 significant miRNAs is shown in Figure 2.

MiRNA capacity for class recognition was tested with the K-nearest neighbors algorithm. Thus, 120 out of 470 miRNAs were identified that classified the eight subclasses of lymphomas (correct classification rate: $86.4 \%$ ) (Supplementary Table S1). In all, 95 of these miRNAs coincide with those identified as significantly differentially expressed by SAM analysis (in blue in Table 1).

The miRNA signatures found by SAM analysis for each lymphoma type are described in more detail below.

Burkitt lymphoma. In all, $12 \mathrm{BL}$ cases were analyzed. The majority of them (11/12) showed C-MYC translocation. A total of 35 (14 upregulated, 21 downregulated) miRNAs were deregulated in $\mathrm{BL}$ compared with the other NHLs. Interestingly, members of the miR-17-92 cluster (miR-17-3p, miR-18a, mir-19a, miR-19b and miR92) were upregulated in BL. Among the downregulated miRNAs, we found the let-7 family miRNAs that are commonly lost in different neoplasias, ${ }^{20}$ miR-155, miR-146a and others already described as lost in $\mathrm{BL}^{24}$ and the miR-29 family ( $\mathrm{a}, \mathrm{b}$ and $\mathrm{c}$ ), which regulates $\mathrm{p} 53 .^{25}$
Chronic lymphocytic leukemia. In all, 14 miRNAs were upregulated and 46 downregulated in CLL samples. MiR-197, which regulates the tumor suppressor gene FUS1, ${ }^{26}$ was the most highly expressed miRNA in these samples. MiR-595 and miR-483 were also upregulated, as has been noted in Wilm's tumor. ${ }^{27}$ Among the downregulated miRNAs, we found miR-15a and miR-16, already described in CLL, where the loss of the 13q14 minimally deleted region induces a clonal lymphoproliferative disorder that recapitulates the spectrum of CLL-associated phenotypes observed in humans. ${ }^{7}$ Other downregulated miRNAs were miR-182, miR-199a*(5p), the let-7 family, miR-424, miR-10a, miR-7, mir-126 and miR-218, whose loss of expression is related with the activation of multiple survival pathways in various cancer models. ${ }^{28}$

Diffuse large B-cell lymphoma. No significant differential miRNA expression was found in this type of lymphoma by SAM analysis. This could be due to the intrinsic heterogeneity of DLBCL cases as an entity, which probably dilutes the miRNA expression differences with other types of lymphomas. This heterogeneity is reflected by the Tnasas web resource class predictor analysis results, where the DLBCL group has the highest error rate $(27 \%$; Supplementary Table S1). Indeed, gene expression profiling data in the series of DLBCL confirm this heterogeneity, revealing that 60 and $40 \%$ of the cases are ABC type and GC type, respectively, according to the classifier of Wright et al. ${ }^{14}$ two DLBCL subtypes with specific miRNA signatures categories. ${ }^{13,29}$

Follicular lymphoma. Two significantly upregulated miRNAs, miR-138 and miR-9 (5p and 3p), were found. Increased expression of miR-9 was previously described in FL samples. ${ }^{11}$ MiR-9, which is activated by MYC, has been shown to regulate nuclear factor- $\kappa B^{30}$ Overexpression of miR-9 or let-7a reduces PRDM1/BLIMP1 levels, ${ }^{31}$ a finding of potential interest in FL cases, characterized by the tightly regulated expression of BCL6 and PRDM1/BLIMP1.

Marginal zone lymphoma/MALT. Three out of eight miRNAs significantly upregulated in MALT lymphoma cases studied belong to the miR-200 family (miR-200a, b and c). These miRNAs are components of two clusters, miR-200a/200b/429 on chromosome $1 \mathrm{p} 36.33$ and miR-200c/141 on chromosome 12p13.31. The other miRNAs located in these clusters, miR-429 and miR-141, were also upregulated. The miR-200 family inhibits the initiating step of metastasis, the epithelial-mesenchymal transition (EMT), by maintaining the epithelial phenotype through directly targeting the transcriptional repressors of E-cadherin, ZEB1 and ZEB2. ${ }^{32}$ The only miRNA in MALT lymphoma cases downregulated relative to the other types was miR-126* (corresponding to miR-126-5p), an miRNA whose expression distinguishes acute myeloid leukemia cases with common translocations. ${ }^{33}$

Mantle cell lymphoma. Only miR-126* was downregulated, as in the case of MALT. Conversely, there were eight upregulated miRNAs, for example, miR-182 and miR-183, which are upregulated in colorectal cancer, ${ }^{34}$ and miR-200c, which was also upregulated in MALT lymphoma cases. Downregulation of miR-126* and upregulation of miR-181c, miR-182, miR-363, miR-654 and miR-768-5p were also found in our previous study after comparing MCL cases with non-tumoral controls (tonsils and lymph nodes). In the same study, miR-182 was also upregulated in MCL cell lines compared with mantle cell controls. ${ }^{17}$

Nodal marginal zone lymphoma. Two miRNAs, miR-370 and miR513, were downregulated in NMZL cases. MiR-370 has been shown to be downregulated in gastrointestinal stromal tumors with $14 q$ loss $^{35}$ and upregulated in acute myeloid leukemia patients with $t(15 ; 17) .{ }^{36}$ In all, 61 miRNAs were upregulated relative to the whole series. The most highly expressed miRNA in this series was miR-150, followed by miR-26b. MiR-150 regulates the expression of the transcription factor c-myb, and plays a key role in B-cell 
Table 1. Differentially expressed miRNAs in each type of lymphoma compared with the rest of samples

\begin{tabular}{|c|c|c|c|c|c|c|c|c|c|}
\hline & miRNA & $\overline{B L}$ & CLL & $\overline{\mathrm{DLBCL}}$ & $\overline{F L}$ & MALT & $\mathrm{MCL}$ & NMZL & SMZL \\
\hline 1 & hsa-let-7a & -1.54 & $\begin{array}{l}-2.24 \\
\end{array}$ & - & - & - & - & 2.23 & - \\
\hline 2 & hsa-let-7c & - & - & - & - & - & - & 1.86 & - \\
\hline 3 & hsa-let-7d & -1.53 & -2.16 & - & - & - & - & 1.87 & - \\
\hline 4 & hsa-let-7e & -1.70 & -2.07 & - & - & - & - & 1.84 & - \\
\hline 5 & hsa-let-7f & -1.67 & -2.70 & - & - & - & - & 2.48 & - \\
\hline 6 & hsa-let-7g & -1.90 & -2.01 & - & - & - & - & 2.55 & - \\
\hline 7 & hsa-let-7i & - & - & - & - & - & - & 1.63 & - \\
\hline 8 & hsa-miR-1 & - & - & - & - & - & - & 1.96 & - \\
\hline 9 & hsa-miR-100 & - & -2.02 & - & - & - & - & 2.07 & - \\
\hline 10 & hsa-miR-106a & - & -1.53 & - & - & - & - & - & - \\
\hline 11 & hsa-miR-107 & - & -1.53 & - & - & - & - & - & - \\
\hline 12 & hsa-miR-10a & -1.62 & -2.58 & - & - & - & - & 1.91 & - \\
\hline 13 & hsa-miR-10b & -1.67 & -2.11 & - & - & - & - & 2.60 & - \\
\hline 14 & hsa-miR-125a & - & -1.68 & - & - & - & - & 1.69 & - \\
\hline 15 & hsa-miR-125b & - & - & - & - & - & - & 1.82 & - \\
\hline 16 & hsa-miR-126 & - & -2.27 & - & - & - & - & 2.33 & - \\
\hline 17 & hsa-miR-126* & - & -1.51 & - & - & -1.58 & -2.26 & 2.53 & 1.79 \\
\hline 18 & hsa-miR-127 & - & - & - & - & - & - & - & 1.56 \\
\hline 19 & hsa-miR-128b & - & - & - & - & - & - & 1.52 & - \\
\hline 20 & hsa-miR-130b & 1.61 & - & - & - & - & - & - & - \\
\hline 21 & hsa-miR-133a & - & - & - & - & - & - & 1.59 & - \\
\hline 22 & hsa-miR-133b & - & - & - & - & - & - & 1.55 & - \\
\hline 23 & hsa-miR-136 & - & - & - & - & - & - & - & 2.00 \\
\hline 24 & hsa-miR-138 & - & - & - & 2.07 & - & - & - & - \\
\hline 25 & hsa-miR-139 & - & - & - & - & - & - & 1.62 & 2.94 \\
\hline 26 & hsa-miR-141 & - & - & - & - & 1.54 & - & - & -1.83 \\
\hline 27 & hsa-miR-143 & - & -1.75 & - & - & - & - & - & - \\
\hline 28 & hsa-miR-144 & - & - & - & - & - & - & - & 3.22 \\
\hline 29 & hsa-miR-146a & -1.79 & -1.67 & - & - & - & - & - & - \\
\hline 30 & hsa-miR-146b & - & - & - & - & - & - & 1.74 & - \\
\hline 31 & hsa-miR-148a & - & -1.56 & - & - & 1.51 & - & - & - \\
\hline 32 & hsa-miR-148b & - & - & - & - & - & - & 1.64 & - \\
\hline 33 & hsa-miR-150 & -2.98 & - & - & - & - & - & 2.86 & - \\
\hline 34 & hsa-miR-152 & - & - & - & - & - & - & 1.57 & - \\
\hline 35 & hsa-miR-155 & -2.12 & - & - & - & - & - & 1.59 & - \\
\hline 36 & hsa-miR-15b & - & -1.80 & - & - & - & - & 1.84 & - \\
\hline 37 & hsa-miR-16 & - & -1.76 & - & - & - & - & 1.88 & - \\
\hline 38 & hsa-miR-17-3p & 1.66 & - & - & - & - & - & - & - \\
\hline 39 & hsa-miR-17-5p & - & -1.55 & - & - & - & - & - & - \\
\hline 40 & hsa-miR-181c & - & - & - & - & - & 1.60 & - & - \\
\hline 41 & hsa-miR-182 & - & -2.90 & - & - & - & 1.71 & - & - \\
\hline 42 & hsa-miR-183 & - & - & - & - & - & 1.61 & - & - \\
\hline 43 & hsa-miR-18a & 1.90 & - & - & - & - & - & - & - \\
\hline 44 & hsa-miR-18a* & 1.66 & - & - & - & - & - & - & - \\
\hline 45 & hsa-miR-191 & - & - & - & - & - & - & 1.70 & - \\
\hline 46 & hsa-miR-192 & - & -1.55 & - & - & - & - & 2.00 & - \\
\hline 47 & hsa-miR-193b & - & -1.96 & - & - & - & - & - & - \\
\hline 48 & hsa-miR-195 & -1.69 & -2.00 & - & - & - & - & 1.86 & - \\
\hline 49 & hsa-miR-197 & 1.81 & 2.93 & - & - & - & - & - & - \\
\hline 50 & hsa-miR-199a* & - & -2.81 & - & - & - & - & 2.19 & - \\
\hline 51 & hsa-miR-199b & - & -2.55 & - & - & 1.69 & - & 1.85 & -2.12 \\
\hline 52 & hsa-miR-19a & 2.05 & - & - & - & - & - & - & - \\
\hline 53 & hsa-miR-19b & 1.69 & - & - & - & - & - & - & - \\
\hline 54 & hsa-miR-200a & - & - & - & - & 2.66 & - & 1.55 & - \\
\hline 55 & hsa-miR-200b & - & - & - & - & 2.68 & - & 1.91 & - \\
\hline 56 & hsa-miR-200c & - & - & - & - & 2.02 & 1.50 & - & -1.64 \\
\hline 57 & hsa-miR-203 & - & - & - & - & 1.75 & - & - & - \\
\hline 58 & hsa-miR-204 & - & - & - & - & - & - & - & 2.02 \\
\hline 59 & hsa-miR-206 & - & 1.81 & - & - & - & - & - & - \\
\hline 60 & hsa-miR-20a & - & -2.12 & - & - & - & - & - & - \\
\hline 61 & hsa-miR-20b & - & -2.08 & - & - & - & - & - & - \\
\hline 62 & hsa-miR-210 & - & - & - & - & - & - & - & -1.90 \\
\hline 63 & hsa-miR-212 & - & - & - & - & - & - & - & 1.53 \\
\hline 64 & hsa-miR-215 & - & - & - & - & - & - & 1.85 & - \\
\hline
\end{tabular}

\begin{tabular}{|c|c|c|c|c|c|c|c|c|c|}
\hline & miRNA & $\mathrm{BL}$ & CLL & DLBCL & $\mathrm{FL}$ & MALT & MCL & NMZL & SMZL \\
\hline 65 & hsa-miR-218 & - & -2.63 & - & - & - & - & 1.64 & - \\
\hline 66 & hsa-miR-221 & - & - & - & - & - & - & 1.63 & - \\
\hline 67 & hsa-miR-223 & - & -1.62 & - & - & - & - & 2.19 & - \\
\hline 68 & hsa-miR-224 & - & -1.77 & - & - & - & - & 1.55 & - \\
\hline 69 & hsa-miR-26a & -1.54 & - & - & - & - & - & 2.08 & - \\
\hline 70 & hsa-miR-26b & -1.92 & -2.11 & - & - & - & - & 2.81 & - \\
\hline 71 & hsa-miR-29a & -1.64 & - & - & - & - & - & 1.89 & - \\
\hline 72 & hsa-miR-29b & -1.74 & - & - & - & - & - & 1.63 & - \\
\hline 73 & hsa-miR-29c & -2.72 & - & - & - & - & - & - & - \\
\hline 74 & hsa-miR-30b & - & - & - & - & - & - & 1.66 & - \\
\hline 75 & hsa-miR-30e-3p & - & - & - & - & - & - & 1.95 & - \\
\hline 76 & hsa-miR-30e-5p & -1.82 & - & - & - & - & - & 1.69 & - \\
\hline 77 & hsa-miR-31 & - & - & - & - & - & - & 1.83 & - \\
\hline 78 & hsa-miR-328 & - & 1.51 & - & - & - & - & - & - \\
\hline 79 & hsa-miR-335 & - & - & - & - & - & - & 1.56 & - \\
\hline 80 & hsa-miR-338 & - & -1.67 & - & - & - & 1.71 & - & - \\
\hline 81 & hsa-miR-340 & - & - & - & - & - & - & 1.54 & - \\
\hline 82 & hsa-miR-34a & - & -1.54 & - & - & - & - & - & - \\
\hline 83 & hsa-miR-34b & - & - & - & - & - & - & 1.50 & - \\
\hline 84 & hsa-miR-363 & -2.12 & -2.59 & - & - & - & 3.26 & - & - \\
\hline 85 & hsa-miR-365 & - & -1.79 & - & - & - & - & 2.11 & - \\
\hline 86 & hsa-miR-370 & - & 1.89 & - & - & - & - & -1.83 & - \\
\hline 87 & hsa-miR-373* & 1.73 & - & - & - & - & - & - & - \\
\hline 88 & hsa-miR-374 & - & -2.14 & - & - & - & - & 2.18 & - \\
\hline 89 & hsa-miR-377 & - & -1.62 & - & - & - & - & - & - \\
\hline 90 & hsa-miR-409-3p & - & - & - & - & - & - & - & 2.11 \\
\hline 91 & hsa-miR-421 & - & - & - & - & - & - & - & 1.99 \\
\hline 92 & hsa-miR-424 & - & -2.68 & - & - & - & - & - & - \\
\hline 93 & hsa-miR-429 & - & - & - & - & 2.16 & - & - & - \\
\hline 94 & hsa-miR-432 & - & 1.97 & - & - & - & - & - & 1.89 \\
\hline 95 & hsa-miR-451 & -1.71 & -2.18 & - & - & - & - & 2.06 & 4.06 \\
\hline 96 & hsa-miR-453 & - & 1.55 & - & - & - & - & - & - \\
\hline 97 & hsa-miR-454-3p & - & -1.54 & - & - & - & - & 1.65 & - \\
\hline 98 & hsa-miR-455 & - & - & - & - & - & - & 1.76 & - \\
\hline 99 & hsa-miR-483 & - & 2.29 & - & - & - & - & - & - \\
\hline 100 & hsa-miR-485-3p & - & 2.39 & - & - & - & - & - & - \\
\hline 101 & hsa-miR-486 & - & - & - & - & - & - & - & 1.56 \\
\hline 102 & hsa-miR-487a & - & - & - & - & - & - & - & 1.71 \\
\hline 103 & hsa-miR-487b & - & - & - & - & - & - & - & 1.99 \\
\hline 104 & hsa-miR-513 & - & - & - & - & - & - & -1.83 & - \\
\hline 105 & hsa-miR-520d & - & 2.00 & - & - & - & - & - & - \\
\hline 106 & hsa-miR-520d* & - & - & - & - & - & - & - & 1.77 \\
\hline 107 & hsa-miR-542-3p & - & - & - & - & - & - & - & 1.89 \\
\hline 108 & hsa-miR-557 & 1.66 & - & - & - & - & - & - & - \\
\hline 109 & hsa-miR-560 & 2.02 & - & - & - & - & - & - & - \\
\hline 110 & hsa-miR-574 & 1.76 & 2.83 & - & - & - & - & - & 1.53 \\
\hline 111 & hsa-miR-595 & - & 2.59 & - & - & - & - & - & 1.95 \\
\hline 112 & hsa-miR-609 & - & 1.51 & - & - & - & - & - & - \\
\hline 113 & hsa-miR-625 & - & -2.82 & - & - & - & - & 2.52 & - \\
\hline 114 & hsa-miR-629 & 2.51 & - & - & - & - & - & - & - \\
\hline 115 & hsa-miR-647 & - & 1.53 & - & - & - & - & - & - \\
\hline 116 & hsa-miR-650 & - & - & - & - & - & - & - & 2.05 \\
\hline 117 & hsa-miR-654 & - & - & - & - & - & 1.67 & - & - \\
\hline 118 & hsa-miR-663 & 3.32 & - & - & - & - & - & - & -1.62 \\
\hline 119 & hsa-miR-7 & - & -2.40 & - & - & - & - & - & - \\
\hline 120 & hsa-miR-766 & - & 1.58 & - & - & - & - & - & - \\
\hline 121 & hsa-miR-768-3p & -1.71 & - & - & - & - & - & 1.95 & - \\
\hline 122 & hsa-miR-768-5p & -1.77 & - & - & - & - & 2.00 & - & - \\
\hline 123 & hsa-miR-9 & - & -2.00 & - & 1.67 & - & - & - & - \\
\hline 124 & hsa-miR-9* & - & -2.69 & - & 1.87 & - & - & - & - \\
\hline 125 & hsa-miR-92 & 1.52 & - & - & - & - & - & - & - \\
\hline 126 & hsa-miR-95 & - & -2.05 & - & - & - & - & 2.22 & 2.64 \\
\hline 127 & hsa-miR-98 & - & -2.24 & - & - & - & - & 2.09 & - \\
\hline 128 & hsa-miR-99a & - & - & - & - & - & - & 2.56 & -2.19 \\
\hline
\end{tabular}

Abbreviations: $B L$, Burkitt lymphoma; CLL, chronic lymphocytic leukemia; DLBCL, diffuse large B-cell lymphoma; FC, fold change; FDR, false discovery rate; FL, follicular lymphoma; KNN, K-nearest neighbors; MALT, mucosa-associated lymphoid tissue lymphoma; MCL, mantle cell lymphoma; miRNA, microRNA; NMZL, nodal marginal zone B-cell lymphoma; SMZL, splenic marginal zone lymphoma. MiRNAs differentially expressed in the different non-Hodgkin lymphoma types. FC in $\log _{2}$ is listed for all miRNAs with FDR $<0.01$ and FC $>1.5$. Numbers in red and green refer to upregulated and downregulated miRNAs, respectively. MiRNAs in blue are those also identified by KNN class prediction. 


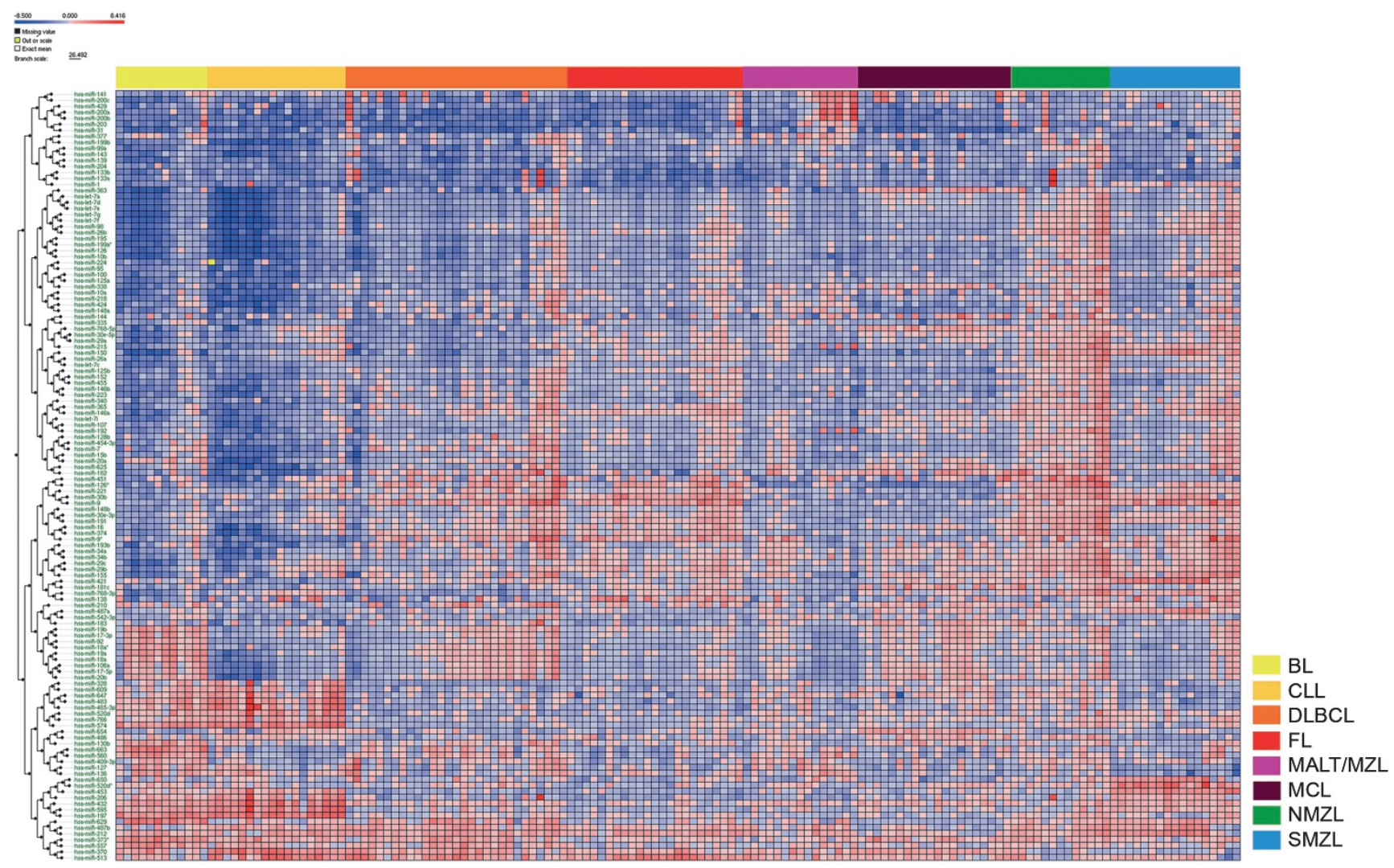

Figure 2. Differentially expressed miRNAs in B-cell lymphomas. In all, 128 miRNAs were differentially expressed in the series (FDR $<0.01$ and fold change $(F C)>1.5$ ) of 147 samples. For representation, data were normalized with non-tumoral controls (tonsils, reactive lymph nodes or spleens).

differentiation. ${ }^{8}$ Interestingly, seven members of the let-7 family of miRNAs, which are commonly lost in tumors, were significantly overexpressed relative to the other lymphoma types.

Splenic marginal zone lymphoma. In all, 26 miRNAs (20 upregulated and 6 downregulated) were differentially expressed in SMZLs. The cluster miR-144/451 is highly overexpressed in SMZL. These two miRNAs are erythropoiesis regulators, ${ }^{37}$ a finding that may be related to the splenic microenvironment. Among the downregulated miRNAs, we found the miR-200c/141 cluster, which was upregulated in other types of B-cell lymphomas in this series (MALT and MCL).

Identification of miRNAs differentially expressed in BL vs DLBCL As a practical application of the data generated here, we compared the DLBCL and BL miRNA signatures. Microarray data from 12 frozen specimens of BL were compared with $29 \mathrm{DLBCL}$ cases using SAM (details of the series, such as C-MYC translocation, $A B C, G C$, subgroups are described in Material and Methods section). In all, 43 miRNAs had a $>1.5$-fold in $\log _{2}$ differential expression and an FDR $<0.01$ (Figure 3 ). These miRNAs were investigated further by qRT-PCR in an additional series of $28 \mathrm{BL}$ and 43 DLBCL FFPE samples.

Six of the miRNAs had low-efficiency qRT-PCR amplification, and were excluded from further analysis. Inefficient amplification could be due to the low quality of the RNA (extracted from FFPE samples), or to their generally low expression level.

Differential expression (FDR <0.05) was confirmed in 19 miRNAs (Table 2 and Figure 4). Thirteen additional miRNAs had the same tendency as observed in microarray analysis, but less significantly. Two miRNAs were significantly expressed, but oppositely with respect to the microarray. So, 32 of 37 miRNAs followed the array tendency (Supplementary Table S2).

MiR-155 was the most significantly lost miRNA in BL, confirming previous findings, ${ }^{23}$ followed by miR-29b and miR-146a, whereas the most significantly lost miRNAs in DLBCL were miR-17-3p, miR-595 and miR-663.

MiR-29b, which is downregulated in BL cases, regulates TCL-1 expression, ${ }^{38}$ a protein that is aberrantly expressed in this type of lymphoma and has been proposed as a diagnostic marker. ${ }^{18,39}$ This miRNA is also negatively correlated with MCL- 1 expression, ${ }^{40}$ a key antiapoptotic protein of the BCL2 family, that is overexpressed in high-grade lymphoid neoplasms.

MiR-146a was already known to play an important role in inflammatory reaction and cancer. ${ }^{41}$ MiR-34b that was downregulated in $\mathrm{BL}$ is targeted by $\mathrm{p} 53$, and is involved in maintaining self-renewal of pancreatic cancer stem cells, possibly by directly modulating the downstream targets BCL2 and NOTCH. ${ }^{42}$

The 19 significant miRNAs were submitted to the SOTA algorithm for samples unsupervised clustering. Only 5 of 71 cases were misplaced (7\%): $4 \mathrm{DLBCL}$ and $1 \mathrm{BL}$. As it is shown in Supplementary Figure S2, DLBCL cases that cluster with BL do not show any common feature in terms of GC/ABC type, C-MYC translocation or BCL2 immunohistochemical expression, although two of the cases carry on a C-MYC translocation. As some of the BL cases in this study do not carry C-MYC translocation, we analyzed C-MYC mRNA and protein expression levels. As shown in Supplementary Figure S3 and Supplementary Table S3 in Supplementary Information, C-MYC expression level does not significantly differ between BL cases with and without C-MYC translocation. On the other hand, the cluster shown in Supplementary Figure S2 demonstrates that these cases do not cluster depending on C-MYC translocation status or C-MYC expression level. 


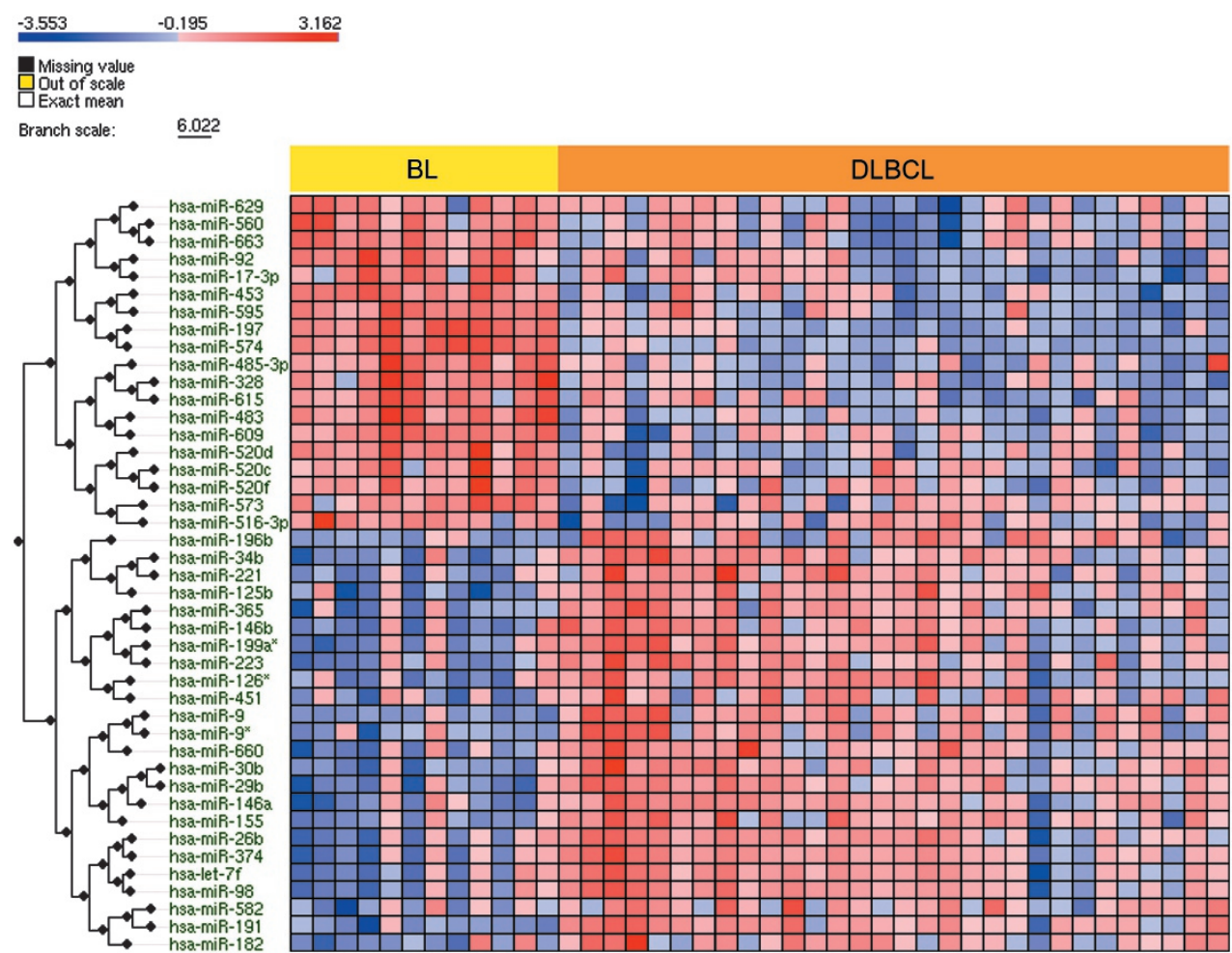

Figure 3. MiRNAs differentially expressed between BL and DLBCL. In all, 43 miRNAs were significantly (FDR $<0.01$ and fold change (FC) $>1.5)$ differentially expressed in a SAM analysis comparing microarray data of $12 \mathrm{BL}$ vs 29 DLBCL.

\begin{tabular}{|c|c|c|c|}
\hline MiRNA & $F D R$ & Average $F C$ & Upregulated in \\
\hline hsa-miR-155 & $<1.00 \mathrm{E}-07$ & 2.67 & DLBCL \\
\hline hsa-miR-29b & $3.00 \mathrm{E}-07$ & 2.33 & DLBCL \\
\hline hsa-miR-146a & $2.30 \mathrm{E}-06$ & 1.97 & DLBCL \\
\hline hsa-miR-17-3p & $2.30 \mathrm{E}-06$ & 1.68 & $\mathrm{BL}$ \\
\hline hsa-miR-365 & $1.75 \mathrm{E}-05$ & 1.59 & DLBCL \\
\hline hsa-miR-30b & $2.92 \mathrm{E}-05$ & 1.21 & DLBCL \\
\hline hsa-miR-595 & $3.74 \mathrm{E}-05$ & 2.79 & $\mathrm{BL}$ \\
\hline hsa-miR-663 & $3.74 \mathrm{E}-05$ & 1.17 & $\mathrm{BL}$ \\
\hline hsa-miR-573 & 0.0003 & 1.73 & $\mathrm{BL}$ \\
\hline hsa-miR-26b & 0.0005 & 0.83 & DLBCL \\
\hline hsa-miR-374 & 0.0037 & 0.70 & DLBCL \\
\hline hsa-miR-520d & 0.0037 & 1.57 & $\mathrm{BL}$ \\
\hline hsa-miR-92 & 0.0037 & 1.27 & $\mathrm{BL}$ \\
\hline hsa-let7f & 0.0076 & 0.67 & DLBCL \\
\hline hsa-miR-516-3p & 0.0096 & 0.83 & $\mathrm{BL}$ \\
\hline hsa-miR-9 & 0.0096 & 1.03 & DLBCL \\
\hline hsa-miR-629 & 0.0170 & 0.88 & $\mathrm{BL}$ \\
\hline hsa-miR-9* & 0.0170 & 1.01 & DLBCL \\
\hline hsa-miR-34b & 0.0502 & 0.80 & DLBCL \\
\hline
\end{tabular}

Abbreviations: BL, Burkitt lymphoma; DLBCL, diffuse large B-cell lymphoma; FC, fold change; FDR, false discovery rate; miRNA, microRNA; qRT-PCR, quantitative real-time-polymerase chain reaction. List of 19 miRNAs differentially expressed between $B L$ and $D L B C L$, confirmed by qRT-PCR: FDR $<0.05$. FC is expressed in $\log _{2}$.

\section{DISCUSSION}

Lymphoma diagnosis is based on the integration of clinical and histopathological data with chromosomal alterations and gene, or protein, expression data. In recent years, there have been many significant advances in lymphoma classification; nevertheless, additional molecular markers enabling a better distinction of specific lymphoma types and a more accurate prediction of response to therapy are still needed. The addition of miRNAs to the diagnostic algorithms is viable, given that qRT-PCR for miRNA expression is feasible in paraffin-embedded tissues, and it has been shown that the miRNA data generated in FFPE samples reproduce the data generated in frozen specimens. ${ }^{13}$ In addition, the identification of miRNAs differentially expressed among lymphoma types could improve our understanding of lymphoma pathogenesis, ultimately enabling recognition of new therapeutic targets.

miRNA target prediction revealed interesting miRNA/gene interactions that could give some light on how lymphomagenesis takes place. For example, miR-133a and miR-23b, which are lost in our series of lymphomas, target PAX5, a gene involved in lymphocyte development and whose upregulation is related to the development of different B-cell lymphoma types reviewed by O'Brien et al. $^{43}$

One of the most strongly lost miRNAs in our series, miR-31, is predicted to regulate the expression of the B-cell receptor pathway together with MAPK and JAK-STAT pathways.

Finally, target prediction tools showed some interesting genes that are commonly lost in different B-cell lymphoma types, and could be targeted by miRNAs upregulated in our series. Therefore, miR-9 and miR-513 are predicted to target PRDM1, and miR-770$5 p$ and miR-212, whose predicted target gene is TNFAIP3. ${ }^{44,45}$

The expression of some of the miRNAs identified here reflects their role in B-cell differentiation, as has been shown for miR-17-5p, miR-20b, miR-223, ${ }^{46}$ miR-150 ${ }^{\text {(ref.8) }}$ and miR- $9 .^{46}$ In fact, the transcription factors LMO2 (GC marker) and PRDM1/BLIMP1 (plasma cell marker), which have a key role orchestrating B-cell differentiation, are found to be targets of some of these miRNAs, ${ }^{46,47}$ which may suggest that the follicular lymphoma GC phenotype may be dependent on the concerted action of multiple miRNAs. Thus, miR-9, which is upregulated in FL cases, is involved in PRDM1/BLIMP1 downregulation, ${ }^{46}$ which is consistent with the downregulation of PRDM1/BLIMP1 observed in reactive and 

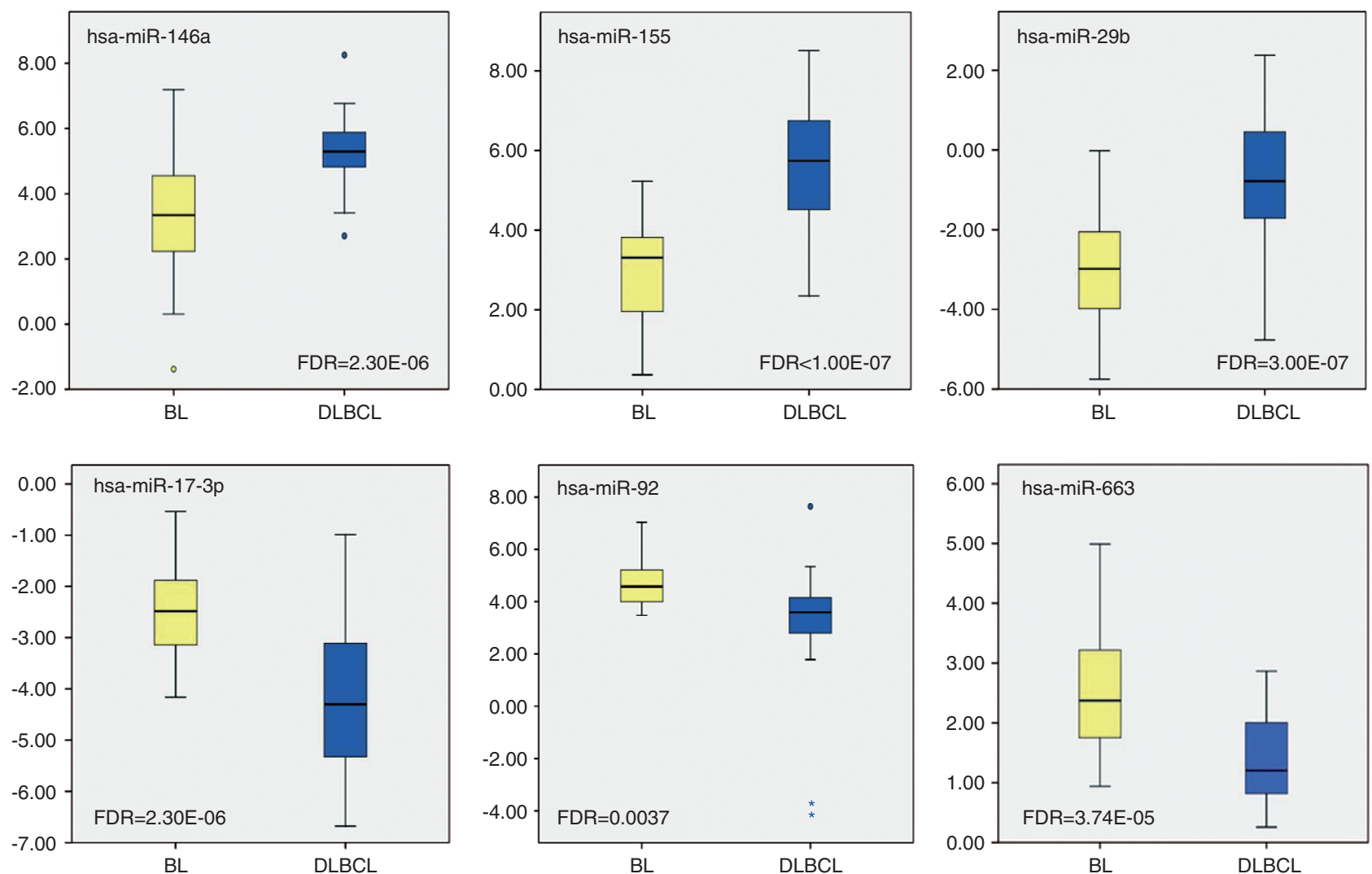

Figure 4. Box plots of $q R T-P C R$ results. A selection of significant miRNAs (BL vs DLBCL) confirmed by qRT-PCR is illustrated. Mir-146a, miR-155 and mir-29b were upregulated in the DLBCL group, whereas miR-17-3p, miR-92 and miR-663 were upregulated in the BL group. $-\triangle C t$ values are represented in $\log _{2}$ scale; ${ }^{*}$ denotes outliers.

neoplastic GC B cells, ${ }^{48}$ thus pointing to a possible cooperation of BCL6 and miR-9 in regulating terminal B-cell differentiation. ${ }^{46}$ MiR223 has been shown to inhibit LMO2 expression, ${ }^{47}$ an observation that correlates with the strong expression of miR-223 in NMZL cases, a tumor that does not express LMO2 or other GC markers. Interestingly, miR-155 expression is downregulated in CLL and upregulated in NMZL cases, which probably reflects the observation that $B$ cells lacking miR-155 generate reduced extrafollicular and $\mathrm{GC}$ responses and fail to produce high-affinity immunoglobulin G1 antibodies, ${ }^{49}$ which is consistent with the data showing that MZL cells have a striking capacity for colonizing GCs and differentiating to become GC cells.

RAG1, BCL6 and PRDM1/BLIMP1 are genes regulated by multiple miRNAs: at least eight miRNAs are predicted to target their 3 '-UTRs, thereby emphasizing the need for the tight regulation of the expression of these transcription factors. MiR-127 is one of the miRNAs already known to regulate BCL6 expression. ${ }^{50}$ Here, it was found to be upregulated in SMZL cases, a tumor that lacks BCL6 expression. ${ }^{1}$ Normal B-cell differentiation and neoplastic B-cell phenotypes seem to be partially determined by the reciprocal antagonism between BCL6 and PRDM1/BLIMP1 expression, ${ }^{51}$ which seems to be at least partially orchestrated through the interaction of multiple miRNAs.

MiR-182, overexpressed in MCL cases, was recently found to be overexpressed in MCL cell lines, ${ }^{17}$ where a role as regulator of FOXO1, a putative tumor suppressor gene downregulated in breast cancer, has been proposed. ${ }^{52}$

Some of the changes detected here reflect the already established heterogeneity of several tumor types. Thus, DLBCL had the most heterogeneous miRNA signature of all the lymphoma types analyzed, extending previous observations. ${ }^{47}$ In fact, heterogeneity of DLBCL has been characterized at the gene expression level, where the distinction between GC GC) type, $A B C$ type and primary mediastinal large B-cell lymphoma has demonstrable biological, prognostic and therapeutic implications. ${ }^{53}$ The series of DLBCL cases studied here reflects this heterogeneity with a $60 \%$ of ABC-type cases and $40 \%$ of GC-type cases in the series used for miRNA profiling. In fact, miRNA signatures related with the molecularly defined subgroups of DLBCL based on the cell of origin have been found by different groups. $^{13,29}$

The heterogeneity is also reflected in C-MYC status; $32.5 \%$ $(13 / 40)$ of FFPE series used for qRT-PCR were positive for C-MYC translocation. This uncommon high percentage of C-MYC translocated DLBCL is due to the fact that this series of DLBCL is a selection of DLBCL cases phenotypically similar to BL (44\% non-GC $(11 / 25)$ and $56 \%$ GC (14/25), as the source of the cases was a collection of DLBCL cases with features mimicking BL submitted for reference diagnosis.

The causes of these miRNA expression gains and losses are still to be investigated in many cases, although some can be attributed to changes in DNA copy numbers in the chromosomal regions where they are located. For instance, analysis of a deletion at $13 q 14.3$ prompted the discovery of two physically linked miRNAs, miR-15a and miR-16-1, which were targets of these deletions, ${ }^{7}$ and shown here to be lost in CLL. NMZL cases exhibited a gain of miR-191 located at chromosome 3p21.31, an area of known gains in NMZL. ${ }^{54}$

The data generated here could have a practical diagnostic value. We have explored whether miRNA data could contribute to the differential diagnosis of $\mathrm{BL}$ and $\mathrm{DLBCL}$, a controversial issue that requires new approaches. ${ }^{3,4,39}$ Despite the characteristic association between C-MYC translocation and the diagnosis of $\mathrm{BL}$, a discrete number of $\mathrm{DLBCL}$ cases may carry the C-MYC 
translocation. ${ }^{1}$ Furthermore, a group of cases with typical histology of BL may lack the C-MYC translocation ${ }^{55}$ and some association with the expression of selected miRNAs has been described. ${ }^{56}$ Our results show that a few miRNAs could be of diagnostic value. For example, BL cases show loss of miR-155 and gain of multiple miRNAs belonging to clusters 17-92. Some of the miRNAs that characterize BL cases are probably C-MYC targets, as has been shown for the clusters 17-92 and other miRNAs. ${ }^{24,57}$ In this study, miRNAs upregulated by C-MYC were upregulated in BL relative to other NHL cases (miR-17-3p, miR-18a, miR-19a, miR92 and miR-130b) and specifically when compared with DLBCL (miR-17-3p). Other miRNAs downregulated by C-MYC were lost in BL compared with other NHL cases (let-7 family, miR-146a and miR-29 family), and some were confirmed when BL was compared with DLBCL (miR-29b and miR-146a). The expression of miR-155 in $B L$ has been controversial, but recent reports agree that BL lacks miR-155 expression, ${ }^{24}$ as confirmed here. In our study, it was the most significantly differentially expressed miRNA in BL compared with DLBCL, with a substantial 2.67-fold $\left(\log _{2}\right)$ change, meaning that expression in DLBCL was around six times stronger than in $\mathrm{BL}$. This confirms miR-155 downregulation in $B L$, making it one of the most suitable markers for differential diagnosis. Nevertheless, a model including all 19 significant miRNAs or the most significant ones may increase the accuracy of the BL diagnosis as the unsupervised cluster failed to classify only $7 \%$ of the samples, and it can also form the basis of a better understanding of BL pathogenesis. These data facilitate a next level of analysis, where an independent series of $B L, D L B C L$ and intermediate $B L / D L B C L$ is currently being studied to obtain a signature with a lower number of miRNAs, which could potentially be used for diagnosis. Furthermore, a recently published paper by Lenze et al. ${ }^{58}$ defines a signature of miRNAs that differentiates BL from DLBCL. Five of the miRNAs described in that paper, expressed in DLBCL, are confirmed by the work presented here, supporting the potential relevance of miRNAs in diagnosis.

Differences in miRNA signatures for different lymphoma types may have multiple causes. As B cells at different stages of maturation show specific miRNA signatures, ${ }^{59}$ the differential expression of miRNAs between different lymphomas types may reflect distinct miRNA profiles of the cell of origin, the normal counterpart for each lymphoproliferative disorder. Alternatively, these differences could be due to the presence of some acquired recurrent genetic alteration of the specific lymphoma type, for example, miRNAs contained in frequently lost chromosomal regions, such as $13 q 14$ deletion in CLL or $7 q 21$ lost in SMZL. In addition, some of the changes observed here could depend on oncogenic changes in genes regulating the expression of multiple miRNAs, as demonstrated for C-MYC and BCL6.

In the initial series of 147 samples, K-nearest neighbors analysis correctly classified $86.4 \%$ of the samples. This rate might be even higher if more cases were introduced. Even though miRNA involvement in the pathogenesis of each specific lymphoma type still needs to be explored, and validation in an independent series of samples is recommended, the miRNA expression signatures described here could be a useful additional tool enabling a more accurate B-cell NHL diagnosis, in particular when BL vS DLBCL differential diagnosis is required, and a better understanding of B-cell lymphoma pathogenesis.

\section{CONFLICT OF INTEREST}

The authors declare no conflict of interest.

\section{ACKNOWLEDGEMENTS}

This work was supported by grants from the AECC, Fondo de Investigaciones Sanitarias (RETICS, PI051623, PI052800); (FI08/00038, LDL), (CP06/00002; NM); the Ministerio de Ciencia e Innovación (SAF2008-03871, SAF2007-65957-C02-02),
Fundación la Caixa and the National Institute of Bioinformatics (GGL), Spain. We thank the Spanish National Tumour Bank Network and Socorro María Rodríguez Pinilla (CNIO), the Gregorio Marañon and La Paz Hospitals, Madrid, Spain, the Virgen de la Salud Hospital, Alberto Arribas, Toledo, Spain, Laura Cereceda and Carmen Almaraz, IFIMAV, Santander, Spain, for expert diagnosis, sample retrieval, tumor banking and technical support.

\section{REFERENCES}

1 Swerdlow SH, Campo E, Harris NL, Jaffe ES, Pileri SA, Stein H et al. WHO Classification of Tumours of Haematopoietic and Lymphoid Tissues. IARC Press: Lyon, 2008

2 Aggarwal M, Sanchez-Beato M, Gomez-Lopez G, Al-Shahrour F, Martinez N, Rodriguez $A$ et al. Functional signatures identified in B-cell non-Hodgkin lymphoma profiles. Leuk Lymphoma 2009; 50: 1699-1708.

3 Hummel M, Bentink S, Berger H, Klapper W, Wessendorf S, Barth TF et al. A biologic definition of Burkitt's lymphoma from transcriptional and genomic profiling. N Engl J Med 2006; 354: 2419-2430.

4 Dave SS, Fu K, Wright GW, Lam LT, Kluin P, Boerma EJ et al. Molecular diagnosis of Burkitt's lymphoma. N Engl J Med 2006; 354: 2431 - 2442.

5 Lu J, Getz G, Miska EA, Alvarez-Saavedra E, Lamb J, Peck D et al. MicroRNA expression profiles classify human cancers. Nature 2005; 435: 834-838.

6 Johnson SM, Grosshans H, Shingara J, Byrom M, Jarvis R, Cheng A et al. RAS is regulated by the let-7 microRNA family. Cell 2005; 120: 635-647.

7 Calin GA, Dumitru CD, Shimizu M, Bichi R, Zupo S, Noch E et al. Frequent deletions and down-regulation of micro-RNA genes miR15 and miR16 at 13q14 in chronic lymphocytic leukemia. Proc Natl Acad Sci USA 2002; 99: 15524-15529.

8 Xiao C, Calado DP, Galler G, Thai TH, Patterson HC, Wang J et al. MiR-150 controls B cell differentiation by targeting the transcription factor c-Myb. Cell 2007; 131: $146-159$

9 Mi S, Lu J, Sun M, Li Z, Zhang H, Neilly MB et al. MicroRNA expression signatures accurately discriminate acute lymphoblastic leukemia from acute myeloid leukemia. Proc Natl Acad Sci USA 2007; 104: 19971-19976.

10 Ruiz-Ballesteros E, Mollejo M, Mateo M, Algara P, Martinez P, Piris MA. MicroRNA losses in the frequently deleted region of $7 q$ in SMZL. Leukemia 2007; 21: 2547-2549.

11 Roehle A, Hoefig KP, Repsilber D, Thorns C, Ziepert M, Wesche KO et al. MicroRNA signatures characterize diffuse large B-cell lymphomas and follicular lymphomas. Br J Haematol 2008; 142: 732 -744.

12 Lawrie CH, Soneji S, Marafioti T, Cooper CD, Palazzo S, Paterson JC et al. MicroRNA expression distinguishes between germinal center $B$ cell-like and activated $B$ celllike subtypes of diffuse large B cell lymphoma. Int J Cancer 2007; 121: 1156-1161.

13 Montes-Moreno S, Martinez N, Sanchez-Espiridion B, Diaz Uriarte R, Rodriguez ME, Saez A et al. miRNA expression in diffuse large B-cell lymphoma treated with chemoimmunotherapy. Blood 2011; 118: 1034-1040.

14 Wright G, Tan B, Rosenwald A, Hurt EH, Wiestner A, Staudt LM. A gene expressionbased method to diagnose clinically distinct subgroups of diffuse large $B$ cell lymphoma. Proc Natl Acad Sci USA 2003; 100: 9991 - 9996.

15 lqbal J, Sanger WG, Horsman DE, Rosenwald A, Pickering DL, Dave B et al. BCL2 translocation defines a unique tumor subset within the germinal center B-cell-like diffuse large B-cell lymphoma. Am J Pathol 2004; 165: 159-166.

16 Hans CP, Weisenburger DD, Greiner TC, Gascoyne RD, Delabie J, Ott G et al. Confirmation of the molecular classification of diffuse large B-cell lymphoma by immunohistochemistry using a tissue microarray. Blood 2004; 103: 275-282.

17 Di Lisio L, Gomez-Lopez G, Sanchez-Beato M, Gomez-Abad C, Rodriguez ME, Villuendas $\mathrm{R}$ et al. Mantle cell lymphoma: transcriptional regulation by microRNAs. Leukemia 2010; 24: 1335 -1342.

18 Aggarwal M, Villuendas R, Gomez G, Rodriguez-Pinilla SM, Sanchez-Beato M Alvarez D et al. TCL1A expression delineates biological and clinical variability in B-cell lymphoma. Mod Pathol 2009; 22: 206-215.

19 Peter ME. Let-7 and miR-200 microRNAs: guardians against pluripotency and cancer progression. Cell Cycle 2009; 8: 843-852.

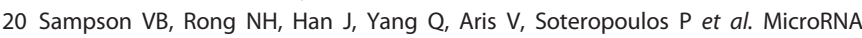
let-7a down-regulates MYC and reverts MYC-induced growth in Burkitt lymphoma cells. Cancer Res 2007; 67: 9762-9770.

21 Gao P, Tchernyshyov I, Chang TC, Lee YS, Kita K, Ochi T et al. c-Myc suppression of $\mathrm{miR}-23 \mathrm{a} / \mathrm{b}$ enhances mitochondrial glutaminase expression and glutamine metabolism. Nature 2009; 458: $762-765$.

22 Lund AH. miR-10 in development and cancer. Cell Death Differ 2010; 17: 209- 214

23 Kluiver J, Poppema S, De Jong D, Blokzijl T, Harms G, Jacobs S et al. BIC and miR155 are highly expressed in Hodgkin, primary mediastinal and diffuse large B cell lymphomas. J Pathol 2005; 207: 243-249. 
24 Robertus JL, Kluiver J, Weggemans C, Harms G, Reijmers RM, Swart Y et al. MiRNA profiling in B non-Hodgkin lymphoma: a MYC-related miRNA profile characterizes Burkitt lymphoma. Br J Haematol 2010; 149: 896-899.

25 Park SY, Lee JH, Ha M, Nam JW, Kim VN. miR-29 miRNAs activate p53 by targeting p85 alpha and CDC42. Nat Struct Mol Biol 2009; 16: 23-29.

26 Du L, Schageman JJ, Subauste MC, Saber B, Hammond SM, Prudkin L et al. miR-93, miR-98, and miR-197 regulate expression of tumor suppressor gene FUS1. Mol Cancer Res 2009; 7: 1234-1243.

27 Veronese A, Lupini L, Consiglio J, Visone R, Ferracin M, Fornari F et al. Oncogenic role of miR-483-3p at the IGF2/483 locus. Cancer Res 2010; 70: $3140-3149$.

28 Gao C, Zhang Z, Liu W, Xiao S, Gu W, Lu H. Reduced microRNA-218 expression is associated with high nuclear factor kappa B activation in gastric cancer. Cancer 2010; 116: 41 - 49 .

29 Jima DD, Zhang J, Jacobs C, Richards KL, Dunphy $\mathrm{CH}$, Choi WW et al. Deep sequencing of the small RNA transcriptome of normal and malignant human B cells identifies hundreds of novel microRNAs. Blood 2010; 116: e118-e127.

30 Guo LM, Pu Y, Han Z, Liu T, Li YX, Liu M et al. MicroRNA-9 inhibits ovarian cancer cell growth through regulation of NF-kappaB1. FEBS J 2009; 276: 5537-5546.

31 Nie K, Gomez M, Landgraf P, Garcia JF, Liu Y, Tan LH et al. MicroRNA-mediated down-regulation of PRDM1/Blimp-1 in Hodgkin/Reed-Sternberg cells: a potential pathogenetic lesion in Hodgkin lymphomas. Am J Pathol 2008; 173: 242-252.

32 Park SM, Gaur AB, Lengyel E, Peter ME. The miR-200 family determines the epithelial phenotype of cancer cells by targeting the E-cadherin repressors ZEB1 and ZEB2. Genes Dev 2008; 22: 894-907.

33 Li Z, Lu J, Sun M, Mi S, Zhang H, Luo RT et al. Distinct microRNA expression profiles in acute myeloid leukemia with common translocations. Proc Natl Acad Sci USA 2008; 105: 15535 - 15540 .

34 Motoyama K, Inoue H, Takatsuno Y, Tanaka F, Mimori K, Uetake H et al. Over- and under-expressed microRNAs in human colorectal cancer. Int J Oncol 2009; 34: $1069-1075$.

35 Haller F, Von Heydebreck A, Zhang JD, Gunawan B, Langer C, Ramadori G et al. Localization- and mutation-dependent microRNA (miRNA) expression signatures in gastrointestinal stromal tumours (GISTs), with a cluster of co-expressed miRNAs located at 14q32.31. J Pathol 2010; 220: 71-86.

36 Dixon-Mciver A, East P, Mein CA, Cazier JB, Molloy G, Chaplin T et al. Distinctive patterns of microRNA expression associated with karyotype in acute myeloid leukaemia. PLoS One 2008; 3: e2141.

37 Rasmussen KD, Simmini S, Abreu-Goodger C, Bartonicek N, Di Giacomo M, Bilbao-Cortes $\mathrm{D}$ et al. The miR-144/451 locus is required for erythroid homeostasis. J Exp Med 2010; 207: 1351 - 1358.

38 Pekarsky Y, Santanam U, Cimmino A, Palamarchuk A, Efanov A, Maximov V et al. Tcl1 expression in chronic lymphocytic leukemia is regulated by miR-29 and miR-181. Cancer Res 2006; 66: 11590-11593.

39 Harris NL, Horning SJ. Burkitt's lymphoma-the message from microarrays. N Engl J Med 2006; 354: 2495-2498.

40 Mott JL, Kobayashi S, Bronk SF, Gores GJ. Mir-29 regulates Mcl-1 protein expression and apoptosis. Oncogene 2007; 26: 6133-6140.

41 Li L, Chen XP, Li YJ. MicroRNA-146a and human disease. Scand J Immunol 2010; 71: $227-231$.

42 Ji Q, Hao X, Zhang M, Tang W, Yang M, Li L et al. MicroRNA miR-34 inhibits human pancreatic cancer tumor-initiating cells. PLoS One 2009; 4: e6816.

43 O'brien P, Morin Jr P, Ouellette RJ, Robichaud GA. The Pax-5 gene: a pluripotent regulator of B-cell differentiation and cancer disease. Cancer Res 2011; 71: $7345-7350$.
44 Malynn BA, Ma A. A20 takes on tumors: tumor suppression by an ubiquitin-editing enzyme. J Exp Med 2009; 206: 977 -980.

45 Nie K, Zhang T, Allawi H, Gomez M, Liu Y, Chadburn A et al. Epigenetic down-regulation of the tumor suppressor gene PRDM1/Blimp-1 in diffuse large B cell lymphomas: a potential role of the microRNA let-7. Am J Pathol 2010; 177: $1470-1479$.

46 Zhang J, Jima DD, Jacobs C, Fischer R, Gottwein E, Huang G et al. Patterns of microRNA expression characterize stages of human B-cell differentiation. Blood 2009; 113: $4586-4594$

47 Malumbres R, Sarosiek KA, Cubedo E, Ruiz JW, Jiang X, Gascoyne RD et al. Differentiation stage-specific expression of microRNAs in B lymphocytes and diffuse large B-cell lymphomas. Blood 2009; 113: 3754-3764.

48 Garcia JF, Roncador G, Sanz Al, Maestre L, Lucas E, Montes-Moreno S et al. PRDM1/ BLIMP-1 expression in multiple B and T-cell lymphoma. Haematologica 2006; 91 : $467-474$.

49 Vigorito E, Perks KL, Abreu-Goodger C, Bunting S, Xiang Z, Kohlhaas S et al. MicroRNA-155 regulates the generation of immunoglobulin class-switched plasma cells. Immunity 2007; 27: 847-859.

50 Saito $Y$, Liang G, Egger G, Friedman JM, Chuang JC, Coetzee GA et al Specific activation of microRNA-127 with downregulation of the proto-oncogene BCL6 by chromatin-modifying drugs in human cancer cells. Cancer Cell 2006; 9: $435-443$.

51 Tunyaplin C, Shaffer AL, Angelin-Duclos CD, Yu X, Staudt LM, Calame KL. Direct repression of prdm1 by Bcl-6 inhibits plasmacytic differentiation. J Immunol 2004; 173: $1158-1165$

52 Guttilla IK, White BA. Coordinate regulation of FOXO1 by miR-27a, miR-96, and miR-182 in breast cancer cells. J Biol Chem 2009; 284: 23204-23216.

53 Dunleavy K, Pittaluga S, Czuczman MS, Dave SS, Wright G, Grant N et al. Differential efficacy of bortezomib plus chemotherapy within molecular subtypes of diffuse large B-cell lymphoma. Blood 2009; 113: 6069-6076.

54 Ferreira Bl, Garcia JF, Suela J, Mollejo M, Camacho FI, Carro A et al. Comparative genome profiling across subtypes of low-grade B-cell lymphoma identifies type-specific and common aberrations that target genes with a role in B-cell neoplasia. Haematologica 2008; 93: 670-679.

55 Leucci E, Cocco M, Onnis A, De Falco G, Van Cleef $P$, Bellan C et al. MYC translocation-negative classical Burkitt lymphoma cases: an alternative pathogenetic mechanism involving miRNA deregulation. J Pathol 2008; 216: $440-450$.

56 Li C, Kim SW, Rai D, Bolla AR, Adhvaryu S, Kinney MC et al. Copy number abnormalities, MYC activity, and the genetic fingerprint of normal B cells mechanistically define the microRNA profile of diffuse large B-cell lymphoma. Blood 2009; 113: $6681-6690$.

57 Chang TC, Yu D, Lee YS, Wentzel EA, Arking DE, West KM et al. Widespread microRNA repression by Myc contributes to tumorigenesis. Nat Genet 2008; 40: 43-50.

58 Lenze D, Leoncini L, Hummel M, Volinia S, Liu CG, Amato T et al. The different epidemiologic subtypes of Burkitt lymphoma share a homogenous micro RNA profile distinct from diffuse large B-cell lymphoma. Leukemia 2011; 25: $1869-1876$.

59 Kuchen S, Resch W, Yamane A, Kuo N, Li Z, Chakraborty T et al. Regulation of microRNA expression and abundance during lymphopoiesis. Immunity 2010; 32: $828-839$.

\section{(c)}

This work is licensed under the Creative Commons AttributionSOMERIGHISRESERVED NonCommercial-No Derivative Works 3.0 Unported License. To view a copy of this license, visit http://creativecommons.org/licenses/by-nc-nd/3.0/

Supplementary Information accompanies the paper on Blood Cancer Journal website (http://www.nature.com/bcj) 